\title{
Free-form 2.5D thermoplastic circuits using one-time stretchable interconnections
}

Jan Vanfleteren, Bart Plovie, Yang Yang, Jelle De Smet, Rik Verplancke, Frederick Bossuyt, Herbert De Smet

imec - Ghent University, Gent-Zwijnaarde, Belgium

\begin{abstract}
A technology is presented for the production of soft and rigid circuits with an arbitrary 2.5D fixed shape. The base of this technology is our proprietary technology for elastic circuits with a random shape, in which the elastic thermoset (mostly PDMS) polymer is now replaced by soft or rigid thermoplastic variants. An additional thermoforming step is required to transform the circuit from its initial flat to its final fixed 2.5D shape, but for rigid fixed shape circuits only onetime stretchability of the extensible interconnects is required, relieving the reliability requirements.
\end{abstract}

\section{INTRODUCTION}

In recent years a lot of research has been spent on the development of dynamically deformable electronics and sensor circuits [1-4]. Such circuits are based on the use of extensible or compressible electrical interconnections, embedded in an elastic polymer like PDMS (silicone rubber) or PU (polyurethane). The circuits, shown in literature, also very often have the property, that they can take different shapes, even when no force other than gravity is executed on it. The same circuit can e.g. be in a flat or a folded state. These circuits are soft random shaped under no external stress. There is however also a lot of interest in electronic circuits which are still soft and dynamically deformable, but return to a fixed shape when external forces are removed. An example of such a circuit is a smart lens with embedded electronic circuits. Such a smart lens should be deformable and soft, but should always have a spherical shape, when no forces are executed on it. Such a circuit can hence be categorized as a soft fixed-shape circuit. Even further, such circuits with a fixed shape can even be completely rigid and not meant to be deformed in any way. Hence such circuits have a rigid fixed (but arbitrary) shape. Applications for such 2.5D free-form rigid circuits include new types of light sources, automotive interior parts (e.g. ceilings with LED illumination), non-flat man-machine interfaces with touch sensors in e.g. household appliances, etc., as they are under development in the frame of the EU funded FP7-TERASEL project [5]. In this contribution a technology will be presented for the fabrication of such 2.5D free form rigid or soft fixed-shape type of circuits, based on the technology, which we have developed earlier for soft random-shape circuits.

\section{TECHNOLOGY}

Common features for the three technologies mentioned above are the following :

- Although the circuits finally will have an arbitrary shape, they are produced entirely on flat temporary carriers. This is compatible with practices in PCB (printed circuit board) or semiconductor industry. 
- Interconnection materials used are those found in PCB industry $(\mathrm{Cu}$, typ. 17 or $35 \mu \mathrm{m}$ thick) or in thin-film circuitry (sputtered metals, e.g. $\mathrm{Cu}$ or $\mathrm{Au}$, with a thickness of typically $1 \mu \mathrm{m}$ or less). These interconnections are patterned using normal conventional lithography and wet etching steps.

- On the circuit pads off-the-shelf components are assembled using conventional lead-free soldering or adhesive assembly technologies.

- To transform the flat circuit to an arbitrary shape the circuit must in at least some parts be extensible or compressible. In general the components are rigid, therefore a stretchable circuit is conceived as a number of rigid components or component islands, interconnected by stretchable conductors. Stretchability of the metal interconnections is achieved by shaping them not as straight lines, but as meanders. The concept is shown in Fig.1.

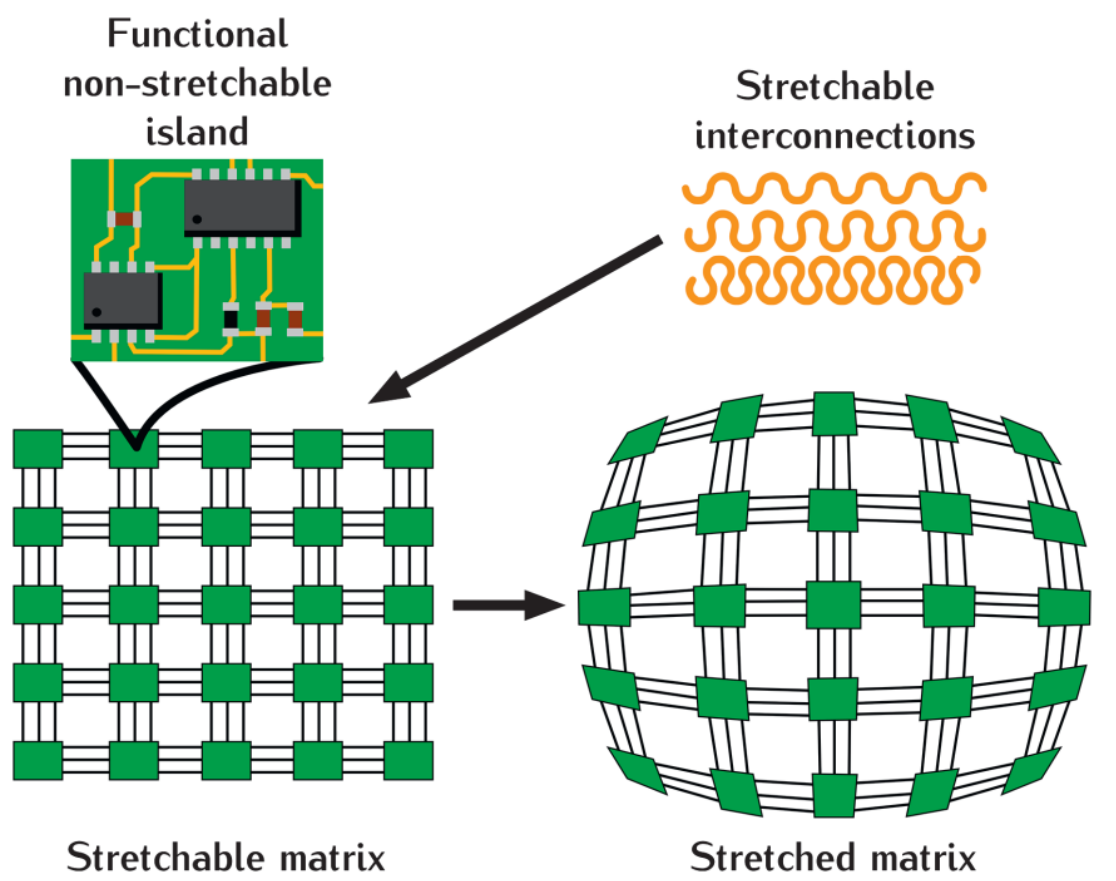

Fig. 1. Stretchable circuit concept: functional non-stretchable component islands, interconnected with electrically conductive, meander shaped stretchable interconnects.

- The circuit is embedded in a polymer or a combination of polymers at the very end of the production process, when all harsh circuit production steps (e.g. metal etching in strong acids, lead-free reflow soldering at temperatures of $250 \mathrm{degC}$ ) have already been executed. This allows us to use a wide variety of polymers, which finally will result in soft random-shaped, soft fixed-shaped or rigid fixed-shaped circuits, depending of the type and thickness of the selected embedding polymer. Fig. 2 depicts the first steps of the process, showing the conventional production and assembly of an electronic circuit on a temporary carrier (e.g. an FR4 or a glass carrier with a wax layer or pressure sensitive adhesive). Fig. 3 shows the 2 -step polymer embedding : after top embedding, the circuit + top polymer layer are released from the temporary carrier. This step is followed by a second polymer application step, realizing the bottom embedding. As an example liquid injection molding of PDMS is shown in Fig.3, but many other polymer processing 
methods and materials can be used (lamination of polyurethanes, PET, polystyrene,...; injection molding of polycarbonate, $\mathrm{ABS}, \ldots ; \ldots$ )

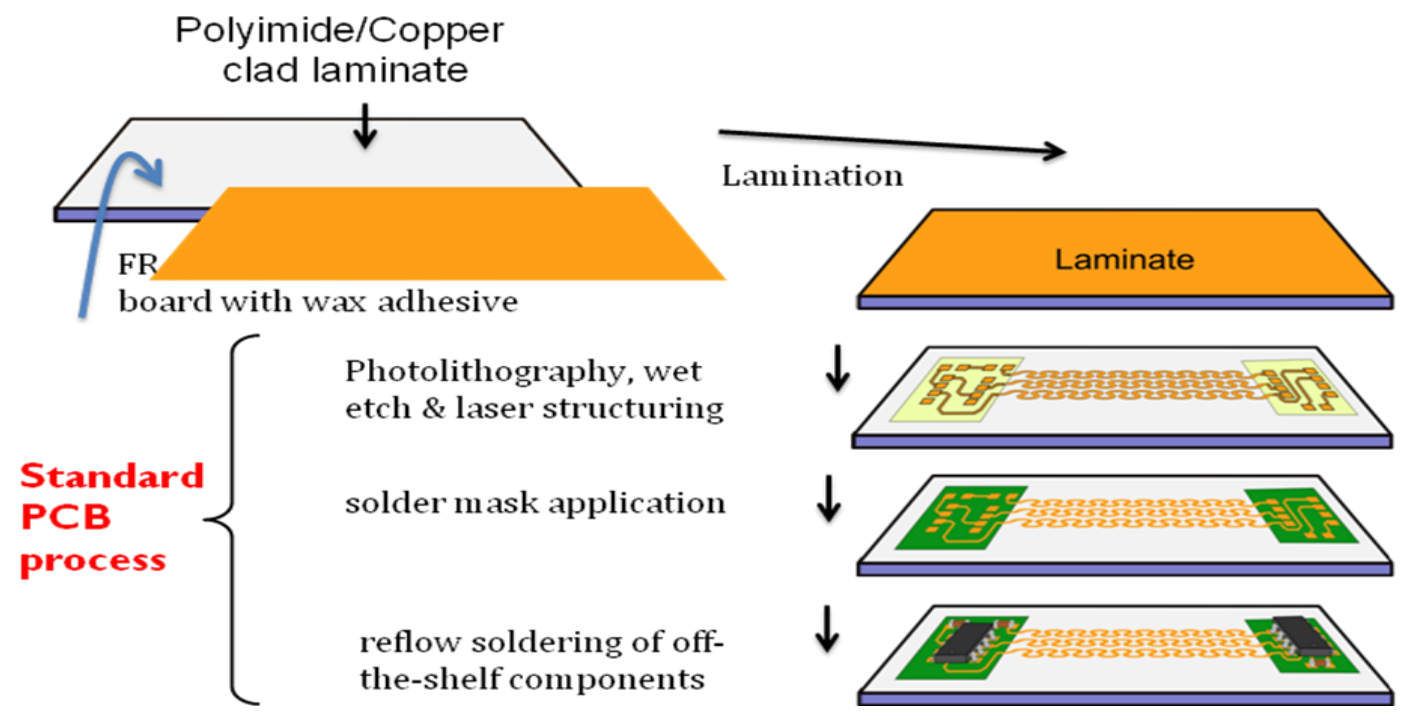

Fig. 2. Circuit production and assembly on a temporary carrier.

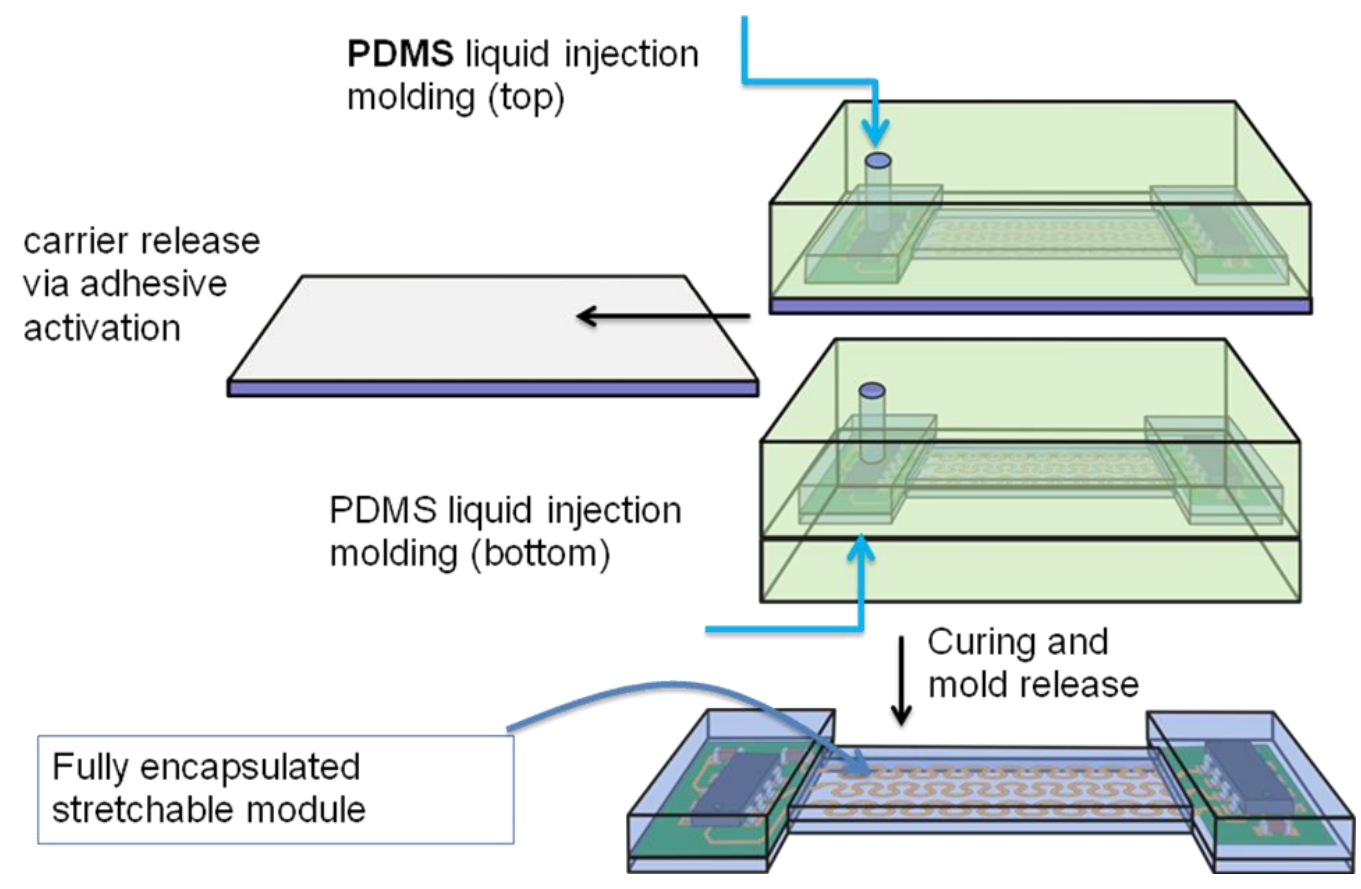

Fig.3. Two-step embedding of the circuit in a polymer and removal of the temporary carrier in between the two steps. As an example PDSM liquid injection molding is shown as the polymer application process.

If the chosen polymer is very soft and is applied with a thickness (e.g. $1 \mathrm{~mm}$ ) which is small compared to the lateral dimensions $(100 \mathrm{~mm}$ or more) of the circuit, the result will be a soft 
random-shape circuit like the one shown in Fig.4. The figure shows a blue light therapy device, containing about 60 LED's, meant for assisting in curing wrist RSI (Repetitive Strain Injury).

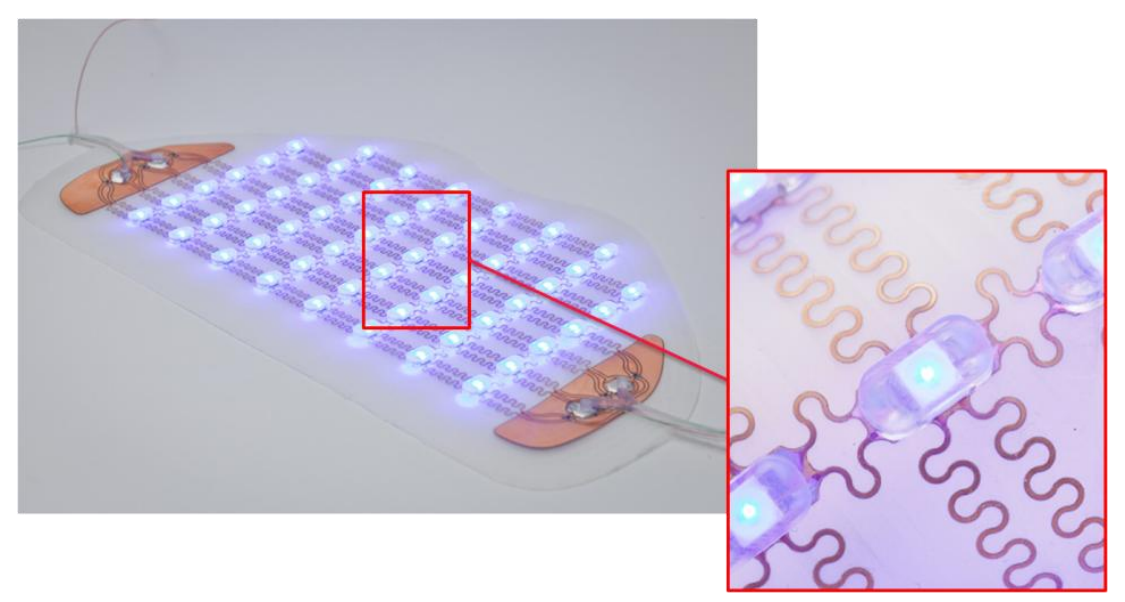

Fig. 4. Soft random-shaped RSI treatment device with approx. 60 blue LED's

In order to produce soft or rigid fixed-shape circuits the polymer embedding step must be complemented with a final step in the production process, which is a thermoforming operation during which the thermoplastic carrier with the embedded circuit is deformed from its original flat shape to the final $2.5 \mathrm{D}$ free form, while heating up the polymer in order to make it deformable with relatively small forces. For a final rigid free form circuit this means that the electrical interconnections should be 1-time stretchable only, in contrast to the large amount of stretch and release cycles which an elastic, dynamically stretchable circuit has to withstand. This single stretch in thermoplastic circuits occurs during the thermoforming step. During this thermoforming process also the components and interconnections inside the carrier material are subjected to an additional thermal and mechanical loading step. A trade-off exists between these 2 loading modes: heating at higher temperatures will reduce the necessary deformation force and vice versa. Heating and forming steps of a thermoforming process are illustrated in figure 5.
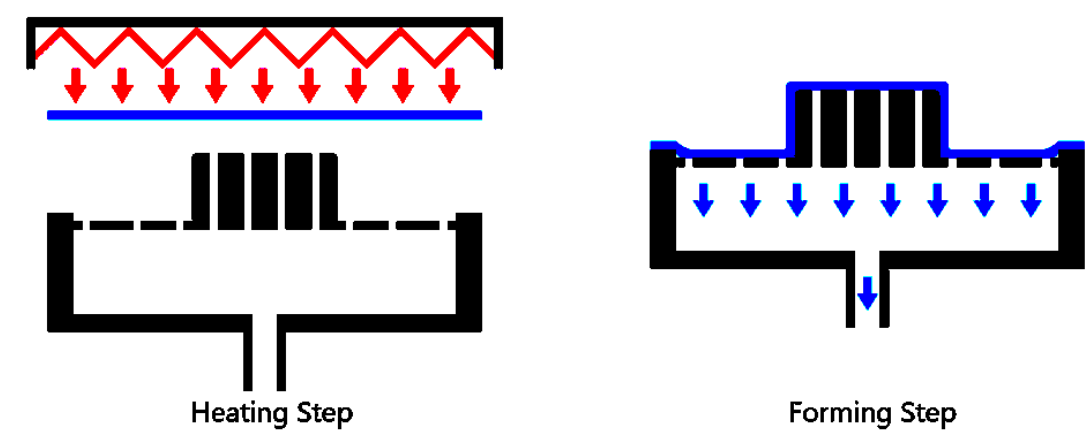

Fig.5 : Thermoforming principle : a flat thermoplastic polymer substrate (represented as a blue line), possibly including an embedded circuit, is heated to or above the softening point (left); the circuit is deformed over a dedicated tool using vacuum (right). Subsequently the circuit is cooled down until it becomes rigid again and removed from the forming tool (not shown). 
First experiments have shown the feasibility of this approach for the fabrication of soft and rigid fixed-shape 2.5D electronics. Fig. 6 shows the result of a first thermoforming experiment, where a (non-optimised) flat circuit is deformed to a semi-sphere. As could be expected the deformation is the largest at the base of the semisphere and nearly zero at the top. Extreme extensions of the meanders at the spots of largest deformation are clearly visible. However the meanders stay intact and no resistance increase could be observed.

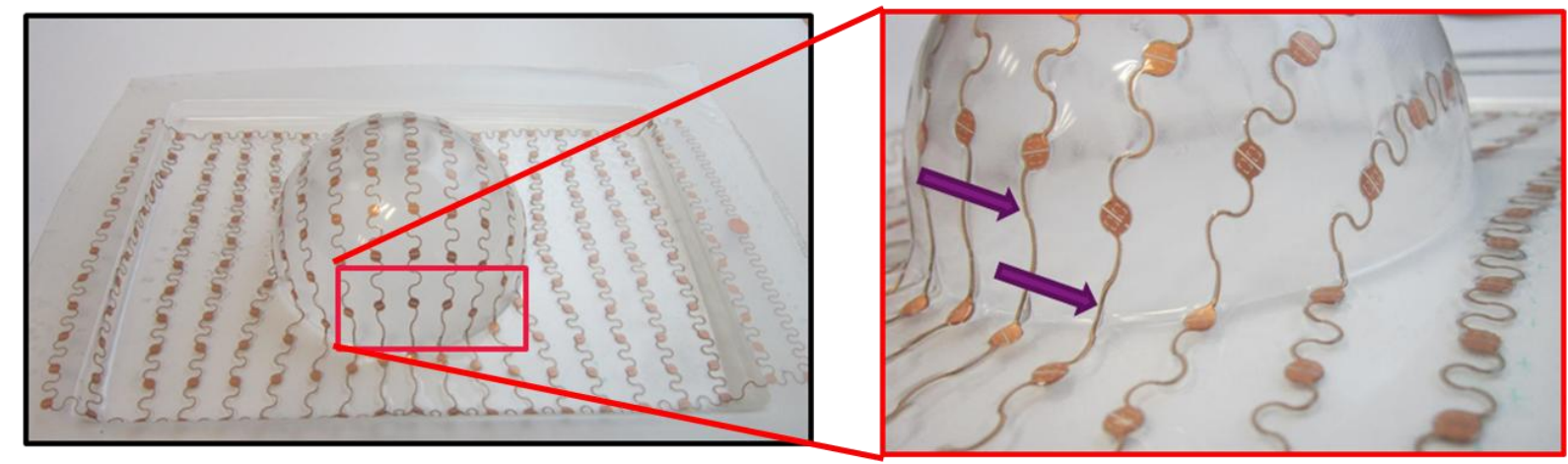

Fig.6 : Thermoforming experiment showing extreme meander elongations at the base of the hemisphere.

Fig 7 shows a functional demonstrator circuit with a more optimized design. A 10-LED flat circuit is embedded in between laminated polycarbonate sheets and subsequently thermoformed. Both meanders and LEDs survive the thermoforming step, resulting in a $2.5 \mathrm{D}$ rigid fixed-shape functional circuit.

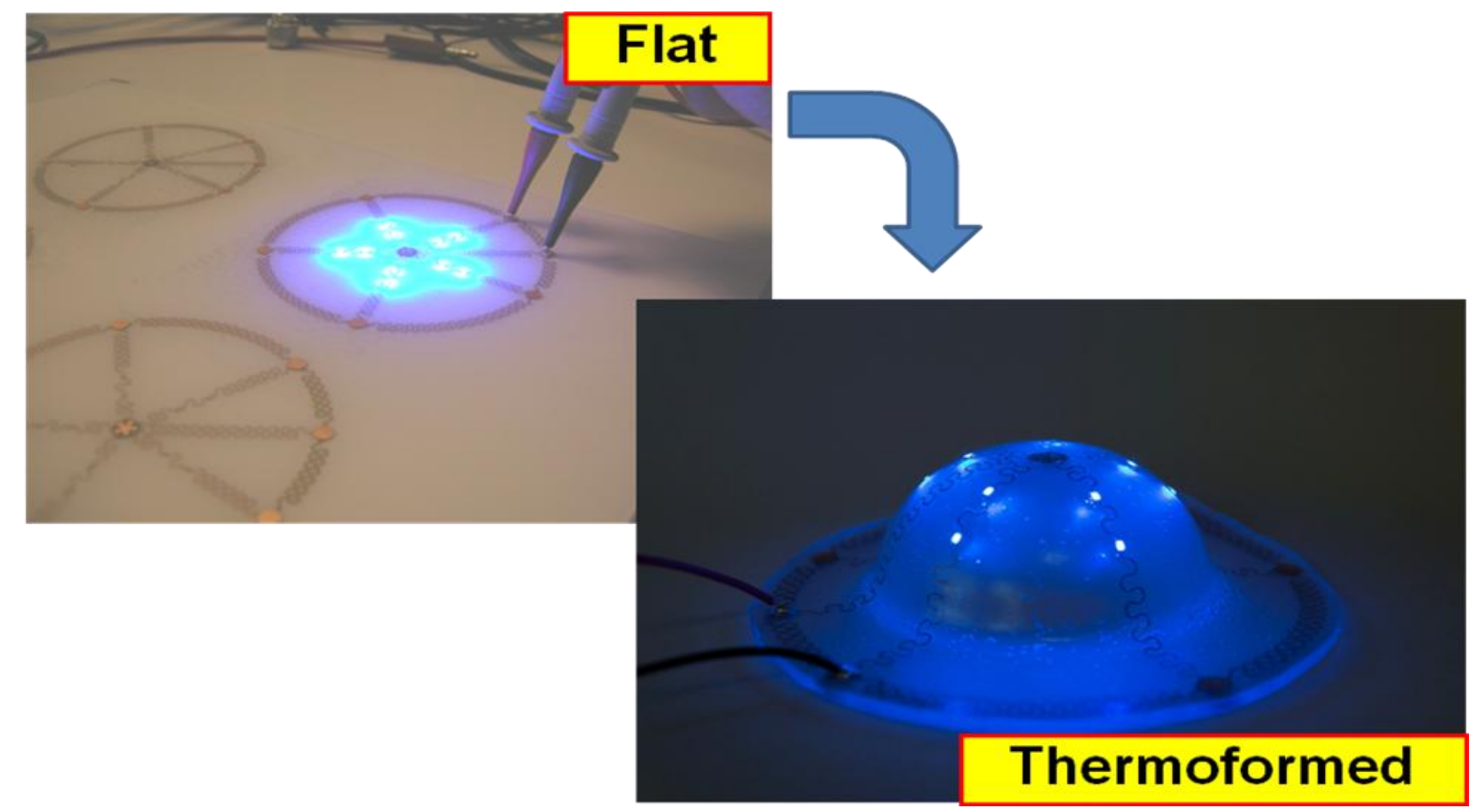

Fig.7 : Thermoforming of a 10-LED flat circuit, embedded in polycarbonate, showing the capability of meanders and components to withstand forming temperatures and pressures. 


\section{CONCLUSIONS AND OUTLOOK}

In initial experiments we have shown the feasibility to produce simple rigid fixed-shape 2.5D electronic circuits. Further work will include modeling of the thermoforming process in order to be able to predict the exact 3D positions of the components and interconnections after the thermoforming step. The final goal of the TERASEL project is to offer a complete value chain for the production of fixed-shape 2.5 electronics circuits. This value chain consists of end users, electronics production and assembly companies and polymer processing companies, all partners in TERASEL.

\section{ACKNOWLEDGMENTS}

The work reported here was carried out in the frame of the European Commission (EC) funded projects FP7-PLACE-It and FP7-TERASEL. We wish to thank the EC for their support.

\section{REFERENCES}

1. T. Sekitani, H. Nakajima, H. Maeda, T. Fukushima, T. Aida, K. Hata, and T. Someya, Nat. Mater. 8, 494 (2009).

2. I.M. Graz, D.P.J. Cotton, A. Robinson, S.P. Lacour, Appl. Phys. Lett. 98, 124101 (2011).

3. D.-H. Kim, J. Song, W.M. Choi, H.-S. Kim, R.-H. Kim, Z. Liu, Y.Y. Huang, K.-C. Hwang, Y. Zhang, and J.A. Rogers, Proc. Natl. Acad. Sci. U.S.A. 105, 18675 (2008).

4. J. Vanfleteren, M. Gonzalez, F. Bossuyt, Y.-Y. Hsu, T. Vervust, I. De Wolf, and M. Jablonski, MRS Bull. 37, 254 (2012).

5. J. Vanfleteren, I. Chtioui, B. Plovie, Y. Yang, F. Bossuyt, T. Vervust, S. Dunphy, B. Vandecasteele, Proc. Technology 15, 208 (2014). 